\title{
Survey on Task Allocation in under Water Vehicle System by using ACO in CNP
}

\author{
Divyanshi Saxena ${ }^{1}$, Miss Priya Sahai ${ }^{2}$ \\ M.Tech Final Year Student, Computer Science and Engineering Department, Babu Banrasi Das University, Lucknow, \\ Uttar Pradesh, India ${ }^{1}$ \\ Asst. Prof, Computer Science and Engineering Dept, Babu Banarsi Das University, lucknow, Uttar Pradesh, India ${ }^{2}$
}

\begin{abstract}
Unmanned underwater vehicle system is a very vast area for research. many organizations are currently working on this. UUV system use contract net protocol to establish the communication. There are many tasks to cover by the one system. This UUV system is design on multi agent based system. In the area of multi agent system contract net protocol is widely used for the establishment of communication between agents. In my research I focus on the working strategy of CNP. There are different areas like genetic algorithm, multi robot task allocation, reservoir flood control system, structural health monitoring, underwater unmanned vehicle system in which CNP works. Although, various algorithms are proposed to improve the efficiency of CNP and they are real effective but there is some area which is untouched for a while. In this paper there is a survey on the interaction protocols on MAS, mainly CNP and also the application area of unmanned underwater vehicles system.
\end{abstract}

Keywords: multi agent system, interaction protocol, contract net protocol, underwater vehicle system.

\section{INTRODUCTION}

There are many technologies exist in the field of multi agent system; which improves the working of MAS in different parameter. MAS exist in area of computer intelligence from more than three decades. Intelligence gives the power to agent to question, learn, act and react. The era of artificial intelligence work as the base for the invention of Multi Agent System. MAS developed to solve to complex problems which could not be solved by using single agent. Agent is nothing but a small computer program or robots which detect the problems and solve them by using its intelligence. Multi agent system is an organization in which there are multiple agents working according to their roles in the system. In many MAS output of one agent is input of another agent. In Multi Agent System there should be autonomous agents as well as collaborative agents established to solve the complex task. This is the main motive to design the multi agent system. MAS are designed to solve the complex or large problem because a single agent is not capable to do that task. [4]

In order to insure the collaboration and cooperation in multi agent system there are various communicative acts of communication language are performed. Communication in MAS is have to be very clear and essential. Communication is the medium which help agents to gain knowledge about environment or surrounding in which they are situated. There are two level of communication in multi agent system- user to agent communication and agent to agent communication. Agent communication with user in order to characterizes their needs and provides them solutions and answers. Communication between agents is established to exchange the resources and knowledge about the task allocated to them. Agent communication language (ACL) is a standard language used by all the agents to communicate with each other. [9] The architecture of multi agent system is designed in a way that they could solve the complex problem, establish communication and other activates like planning, negotiation and decision making. [5]

There are some protocols which are used for interaction in multi agent system. These interaction protocols helps agent to communicate in a proper manner. These protocols are the certain rules of synchronization for communication in multi agent system. Interaction protocol enable agent to exchange message and accomplish communication with other computing agent. The main function of interaction protocol is that each agent in system communicates effectively without having any interference. It is important to ensure that follow the protocol will achieve the goal associated with the final state. Protocol is more efficient with less information needs to be transmitted and less time need to be spent in communication. There are some existing interaction protocols attend by different agents for different functionalities like responding to message, performing an action in their respective field and updating their local state. Interaction with the organization is one of the basic concept of multi agent system, according to Charif Y. ," for agent, interaction with another is both source of its power and source of its problem.." interaction comes from the dynamic linking of several agent through the set of reciprocal actions. Several types of interaction depend on three parameters-: goal, resources and skills. [5] 
There are some existing protocols are-: Coordination protocol, Cooperation protocol, Negotiation protocol, Contact net protocol, Contract net trust establishment protocol.[9]

In this paper we mainly focus on conrract net protocol and underwater vehicle system. This paper is divided into five sections; in first section introduction of the topic, in econd section there is the literacture survey on contract net protocol and underwater vehicle system, in third section there is an application areas of UUV, in forth section there is a conclusion of this paper, and in fifth section the list of references is present.

\section{LITERATURE SURVRY}

First of all we have to discuss about existing interaction protocols. In current time mainly four interaction protocols are in use. Each have different type of functionality. Each have different method for communication. Interaction protocols are use to establish a communication between different agents. in multi agent system each agent has the capability to communicate with all other situated agents, with this ability, agents can interact with their peer to achieve the goals and improve the efficiency of the system [9].During the state of communication whole multi agent system works as a single unit which is known as coherent system. For different type of communication different protocols are used. According to Koji M. and Jin-Hua these protocols are used to define the sequence of message communicated between agents and describe how agent should react to message received during interaction. If an agent negotiate that he works on some specific protocol then that agent is abide by its syntax and semantic rules. These semantic rules behind the protocols should be clear for establishment of proper communication. In multi agent system the interaction between agents is primarily based on two aspects: i.e. degree of heterogeneity means the diversity between the agents and degree of communication means in which manner they are communicating with each other. In the coherent system, some agents are same kind of agents which are known as homogeneous agents and some agents are different in their functionality which are known as heterogeneous agents. Under the one organization there should be unique names of each agents to communicate easily. This helps agents to provide the information about there peer agent and a robust and scalable model of multi agent system can be generated by mediator agent [11]

Various interaction protocols are existed in multi agent system which are separated according to their functionalities; coordination protocol, cooperation protocol, negotiation protocol, contract net protocol, contract net trust establishment protocol.

According to Rashmi Singh, Coordination protocol is implemented among multiple agents to achieve individual goals as well as group goals. Basically to maintain the dependencies between the actions of different kinds of agents, coordination protocol is required. C Coordination between the agents is use to add intelligence in system when no single agent has the enough information about the problem. It I is also use to meet the global constraints. The ANDIOR graphs are used to represent the model of the roles of agents, dependencies between the actions of the agents, and the required recourses to complete the task. [9].

According to Aarti Singh, cooperation protocol, first divide the task into subtask and distribute them all the member agents in the coherent system. This is based on divide and conquers strategy; initially complex task is divided into subtask and then distributed among various agents according to their roles in the system. The main reason of using this protocol is to decrees the complexity of the task and optimization of resources allocation between agents. [9]

According to Saurabh Mukharjii, in negotiation protocol, there are two or more agents involve in this process. With the help of their negotiation joint decision is reached and each agent has their individual goal. To determine the position of agent in the system and the level of agreement of negotiation, the semantics use by agents should be clear. The main features of negotiation are that the language is used by the member agent. In negotiating stage an agent may compromise or deny to compromise and decide to work alone. [9]

CNP is high level protocol which is widely used in the multi agent system, concerned with the clarification of the communication rather than the transmission of bit wise data. It categorizes the agents as either initiator/manager or participant/contractor. For different type of task the agents exchange their roles under one system. Contract net trust establishment protocol is the extended version of CNP which is proposed by Singh \& Juneja in 2010, this protocol states that trust should be established between negotiating agents before the actual communication starts.

Unmanned Underwater Vehicle (UUV) formation system has an important role in the utilization of marine resource. In order to provide an efficient method to research modelling and simulation of UUV formation in the marine environment, the novel approach based on Multi-Agent Interaction Chain was proposed for the UUV formation system. Firstly, Multi-Agent Interaction Chain was analyzed, which mainly considered task and role of UUV in the formation, and the overall modelling process of UUV formation sys- tem based on Multi-Agent Interaction Chain was established. Then the static structure of Multi-Agent based Interaction Chain (MAIC) was researched by focusing on Hybrid UUVAgent model structure. The dynamic mechanism based on Multi-Agent Interaction Chain was created, which was use to focus on collaboration model and interaction model with the help of Adaptive Dynamic Contract Net Protocol.[1] With the development and usage of marine resource and other fields, research on Unmanned Underwater Vehicle (UUV) formation system has become an important technique. There are two advantages of it as follows:[3] 
(1) The UUV possesses the characteristics of good concealment and strong adaptability in under- water, which can complete the task and obtain marine information that humans are not able to.

(2) With the help of UUV formation system the efficiency of task can be improved by collaborating them. Therefore, the UUV formation system has been widely used in marine hydrology survey, marine environmental monitoring, seafloor topographic survey, intelligence reconnaissance, and underwater warfare.

The key properties of UUV formation system are as follows: [7]

(1) The environment of underwater has a great uncertainty which effect the performance of UUV formation.

(2) There are many complex underwater tasks which are needed to complete by UUV, like formation forming, collaborative search and avoiding obstacle.

(3) The capacity of a single UUV is not enough to solve the complex tasks alone so that distributed structure of UUV is presented which shows the high level capacity.

(4) UUV has the power to encapsulate the knowledge about the actions and roles which helps it to perform simple actions of underwater automatically.

(5) Underwater six degrees of freedom movement is accomplish by UUV through mutual collaboration of the different parts of UUV like sensor, controller and actuator. This show that UUV has complex internal structure.

Given that the UUV formation system has almost all the features of a complex system, the perspective of complexity modelling has become an important research. So, traditional complex system simulation and modelling methods such as physical model, mathematical model and graphic model cannot adapt to the simulation and modelling of UUV formation system.

\section{APPLICATION AREAS}

\section{A. Contract Net Protocol}

According to Tuomas Sandholm this is high level protocol and widely used by agents to communicate. The contract net protocol (smith 1980; Smith \& Davis 1981) in distributed artificial intelligence is one of the important paradigms for decentralized allocation of task. Two main agents are participates in CNP; one is manager and other one is contractor. He discussed the implementation of contract net protocol based on marginal cost calculation. The formal model is present by Davis and Smith in which the marginal cost of the task preformed, is locally calculated by the agent itself. The extended version of this framework is use to handle task interaction. These tasks are clustered in the set of task which are going to negotiate.

Gina Bullock and team proposed a structural health monitoring system by using contract net protocol. [4] According to them CNP is task sharing protocol for a collection of software agents that forms a contract net. It was develop to specify problem solving communications and control for nodes in high level distributed problem solving protocol by negotiating process. They also states that each agent in the system for different time or different task can be contractor or manager.

Sandeep kaur and his team present in their journal the updated version of CNP.[10] In this he states that if there are any changes made into the task which is allocated to the contractor agent by the manager agent can be modified during the processing of task before the final report of completion is send by contractor agent. This saves the overhead of restating the process of CNP between the processing agents who wish to coordinate to in order to achieve the some certain objectives. This helps to improve the efficiency and effectiveness of protocol in case if there is a frequent change in set of requirements of manager agents.

Hongtao Liang and Fengju kang states that rapid development of marine resources exploitation, unmanned underwater vehicle as a underwater vehicle robot has been widely used in many marine field UUV as an underwater vehicle robot can carry different sensors to complete underwater mission through high precision and high efficiency motion control under unknown and complex underwater environment.[3]

In their next journal they proposed a improved contract net protocol by using regional trust degree. ICNP is mainly focus on multi stage reducing network communication to improve the efficiency of the system and the quality of task completed. Mainly they propose a prototype model for UUV swarm system, in which ICNP is used to improve the collaborative capability and efficiency of the system.[1]

\section{B. underwater vehicle system}

Oceanography covers a wide range of task loke marine life and ecosystem, ocean circulation, plate tectonics and geology of sea floor, and the chemical and physical property of the ocean.

Geology of seafloor is use to find out the structure of the surface of the ocean. There are many thing that covered by water like hills, mountains and valleys. Oceanography is use to find out the life underwater.

Intelligence reconnaissance is a mission to obtain the information by visual observation or other detection method, about the activities and resources of an enemy or potential enemy or about the Metrologic, hydrolographic or geographic characteristics of the particular area. 
The tasks covered by intelligence reconnasiance:-

- Mission

- Enemy

- Troops and support avaliable

- Time available

- Civil consideration

\section{CONCLUSION}

This research paper shows the task allocation process in underwater vehicle system is a wide area for research. UUV system brings the drastic changes in many fields like marine hydrology, underwater warfare, oceanography, seafloor survey, and life underwater survey. CNP established the communication in UUV system. Tasks are allocated by different process but each process shows different results. Some methodology decrees the execution time, some methodology increases the accuracy. To establish the communication in UUV system using the CNP is the best. There are many existing methodologies which are used which CNP to improve the performance of it.

\section{REFERENCES}

[1] Hongtao Liang, Fengiu kangin "A novel task optimal allocation based on contract net protocol for agent oriented UUV swarm system modelling" Elservier Optic 127-3938-3933, 2016

[2] Elgarej Mouchine, khalifa Mansoori, Yousefi Mohamed, “An Improved Ant colony optimization using multi agent system" the international journal of multi disciplinary science, Issue-2, Vol-3, ISSN-2421-9606, 2016.

[3] Hongtao Liang, Fengju Kang and Hong Li, "UUV formation system modelling and simulation research based on Multi-Agent Interaction Chain" international journal of modelling, simulation, and scientific computing vol-6, no-2 2015.

[4] Gina Bullock, William Nick, kassahun Asamene, Albert esterline "A multi agent and work flow system for structural health monitoring using the contract net protocol and alternative" North carlina A \& T state university, 2015

[5] M.k.Ahmed, A.A.Obinigi, "Multi agent based patient scheduling using ant colony optimization" IEEE, Vol-8, No-5, 2015.

[6] Dimple Juneja Ankit Jagga, Aarti Singh, "A review of FIPA and standardization of agent communication language and interaction protocols" Journal of netwrk communications amd emerging technologies, vol-5, special issue 2, 2015.

[7] Hongtao Liang, Fengju Kang, "Artifical immune intelligent modelling for UUV Underwater navigation system based on immune multi agent network" International journal of innovative computing, information \& control, vol-11, No-5 2015.

[8] Roger P. Stokey, Lee E. Freitag, "A compact control language of AUV acoustic communication”, woods hole oceanographic institution, 2014.

[9] Rashmi Singh, Aarti Singh, Saurabh mukhergee, "A critical investigation of Agent interaction Protocol in multi agent system" international journal on advancement in technologies, ISSN 0976-4860 2014.

[10] Sandeep Kaur, Harjeet Kaur, Summit Courser, "A modification of contract Net protocol a ruler updation approach" international journal of advanced computer science and application,vol-4, no-11, 2013.

[11] Allksis lienka, egons lavendelis, "Experimental analysis of contract net protocol in multi-robot task allocation" in 2013

[12] Seokhoon Yoon, Abul k. Azad, "An UUV aided underwater routing protocol for underwater acoustic Sensor networks", sensors, ISSN 1424$8220,2012$.

[13] Sorin Ilie and Costin Badica, "Multi-Agent Approach to Distributed Ant Colony Optimization" University of Craiova, software engineering department, 2011.

[14] Wei haung, Xingnam zhang, xiaod obgwei, "An improved contract net protocol with multi agent system for Resevoir flood control dispatch" international journal of water resourses and protection vol-3, 735-746, 2011.

[15] Ozgur YILDIZ, Asim Egemen YILMAZ, Bulent GOKALP, "State-of-the-Art System Solutions for Unmanned Underwater Vehicles" Radio engineering, vol-18, no-4, 2009. 